\title{
OMAE2017-61062
}

\section{A COUPLED CFD/MULTIBODY DYNAMICS ANALYSIS TOOL FOR OFFSHORE WIND TURBINES WITH AEROELASTIC BLADES}

\author{
Yuanchuan Liu \\ Department of Naval \\ Architecture, Ocean and Marine \\ Engineering, University of \\ Strathclyde \\ Glasgow, UK
}

\author{
Qing Xiao \\ Department of Naval \\ Architecture, Ocean and Marine \\ Engineering, University of \\ Strathclyde \\ Glasgow, UK
}

\author{
Atilla Incecik \\ Department of Naval \\ Architecture, Ocean and Marine \\ Engineering, University of \\ Strathclyde \\ Glasgow, UK
}

\begin{abstract}
Aero-elasticity is an important issue for modern large scale offshore wind turbines with long slender blades. The behaviour of deformable turbine blades influences the structure stress and thus the sustainability of blades under large unsteady wind loads. In this paper, we present a fully coupled CFD/MultiBody Dynamics analysis tool to examine this problem. The fluid flow around the turbine is solved using a high-fidelity CFD method while the structural dynamics of flexible blades is predicted using an open source code MBDyn, in which the flexible blades are modelled via a series of beam elements. Firstly, a flexible cantilever beam is simulated to verify the developed tool. The NREL $5 \mathrm{MW}$ offshore wind turbine is then studied with both rigid and flexible blades to analyse the aero-elastic influence on the wind turbine structural response and aerodynamic performance. Comparison is also made against the publicly available data.
\end{abstract}

\section{INTRODUCTION}

In the past few years, offshore wind turbines are becoming larger in size to achieve better power capacity and thus reduce the economic costs. It is reported by WindEurope [1] that the average rated power capacity is $4.8 \mathrm{MW}$ for wind turbines installed during the first half of 2016. For a typical offshore wind turbine with a capacity of $5 \mathrm{MW}$, the rotor diameter is 126 meters [2], for which the aero-elasticity of the slender turbine blade cannot be ignored during the design and operating stages.

The study on the aerodynamic performance and structural response of wind turbines with flexible blades has been done with different analysis tools at various orders of accuracy. The highly efficient and well-known analysis package FAST, developed at NREL [3], employs the Blade Element Momentum method to cope with the aerodynamics of blades, while the blade aero-elasticity is modelled with a modal analysis method which requires blade mode shapes as input. More sophisticated tools involve the coupling of Computational Fluid Dynamics (CFD) and Computational Structural Dynamics (CSD) codes such as [4, 5]. However, these numerical modelling tools are often quite computationally time-consuming. To balance the accuracy and computational time, Pierangelo and Jayanarayanan [6] coupled a CFD solver TURNS with an open source multi-body dynamics (MBD) code MBDyn. The flexibility of blades was modelled with a series of beam elements, which can be justified by the slender characteristics of the turbine blades, similar as in $[7,8]$.

The present study aims to develop a fully coupled fluidstructure interaction (FSI) analysis tool for offshore wind turbine associated with potentially deformable blades. This is achieved by coupling the open source CFD toolbox OpenFOAM with the aforementioned MBD code MBDyn. In particular, the aerodynamic loading estimated by CFD interacted with structural responses obtained from MBDyn via a TCP/IP protocol. To verify the methodology we developed, the passive motion of a flexible cantilever beam in the wake of a square solid body is firstly studied. The NREL (National Renewable Energy Laboratory, Colorado, USA) 5 MW baseline offshore wind turbine [2] with both rigid and flexible blades is then modelled. Comparisons between the present results with other public data are made in the aspects of turbine structural response and aerodynamic performance. This work will serve as the foundation for the near future study on a floating offshore wind turbine with deformable blades.

\section{NUMERICAL METHODS}

In the present study, a fully coupled FSI analysis involves three dedicated parts interplaying with each other, including a CFD solver for fluid flow around the turbine, an MBD solver for structural responses and a mesh motion solver to update the CFD mesh as well as to interface with the flow and structural solvers. 


\section{Flow Solver}

The fluid flow around the turbine is solved with the transient CFD solver pimpleDyMFoam in OpenFOAM, which is ideal for incompressible and turbulent flow of Newtonian fluids with a moving mesh. The fluid flow is governed by the continuity equation and Navier-Stokes equations:

$$
\begin{gathered}
\nabla \cdot \mathbf{U}=0 \\
\frac{\partial \mathbf{U}}{\partial t}+\nabla \cdot\left[\left(\mathbf{U}-\mathbf{U}_{g}\right) \mathbf{U}\right]=-\frac{\nabla p}{\rho}+\nabla \cdot\left[v_{e f f} \nabla \mathbf{U}+(\nabla \mathbf{U})^{T}\right]
\end{gathered}
$$

where $\mathbf{U}$ and $\mathbf{U}_{g}$ represent the velocity of flow field and grid nodes, respectively; $\quad p$ is the pressure of flow field; $\rho$ is the fluid density; $v_{\text {eff }}=v+v_{t}$ denotes the effective kinematic viscosity of fluid, in which $v$ and $v_{t}$ are the kinematic and eddy viscosity respectively. The two-equation $k-\omega$ shear stress transport (SST) turbulence model [9] is employed for the turbulence modelling in this study and wall functions are adopted for near-wall treatment.

The PIMPLE (merged PISO-SIMPLE) algorithm is applied to deal with the velocity-pressure coupling in a segregated way. A second-order Crank-Nicolson scheme is used for the temporal discretisation and a second-order upwind scheme is applied for the convective terms. The gradient terms are discretised with a cell-limited Gauss linear scheme.

\section{Structural Solver}

The dynamic response of the structure is solved using the open source general-purpose MBD code MBDyn. The code adopts a Lagrange multiplier or redundant coordinate set formulation for a multibody system consisting of rigid and flexible bodies connected by kinematic constraints [10]. For each body of the constrained system, Newton-Euler equations of motion are established in differential-algebraic form as a set of first-order equations together with the constraint equation as follows:

$$
\begin{gathered}
\mathbf{M} \dot{\mathbf{x}}=\mathbf{p} \\
\dot{\mathbf{p}}+\phi_{\mathbf{x}}^{\mathrm{T}} \lambda=\mathbf{f}(\mathbf{x}, \dot{\mathbf{x}}, t) \\
\phi(\mathbf{x}, t)=0
\end{gathered}
$$

where $\mathbf{M}$ is the inertia matrix of the body; $\mathbf{x}$ represents the vector of the generalised coordinates including both translational and rotational parameters in the global reference frame; the dot operator above a variable denotes its derivative to time; $\mathbf{p}$ is the momentum of the body; $\phi$ is a set of kinematic constraints applied on the body and $\phi_{\mathrm{x}}^{\mathrm{T}}$ is the Jacobian of $\phi$ with respect to the generalized coordinates $\mathbf{x} ; \mathbf{f}$ is the external force and moment exerted upon the body which might be related to its displacement and velocity as well as time. An implicit multistep integration scheme is applied to solve the equation system.

In order to model flexible bodies in MBDyn, a finite volume multibody beam element is implemented [10]. In this method, a flexible body can be modelled as one or more beam elements, each of which is divided into three beam element portions by two evaluation points indicated as squares in Figure 1. The three beam element portions are associated with three reference points (circles), which represents the elastic axis of the beam. Those reference points do not necessarily need to be on a straight line and can be offset from the geometrical nodes (triangles), where equilibrium equations are established considering both external and internal forces as well as moments. External forces and moments are integrated over every beam element portion related to a reference point and later translated to a corresponding node. Meanwhile, internal forces and moments are evaluated at the cross sections of the evaluation points and they are related to the geometrical strains and curvatures via the constitutive laws specified by users. A system of equilibrium equations, i.e. Eq. (3)-(5), is then established and solved for all geometrical nodes, representing either a rigid body or a component of a beam element.

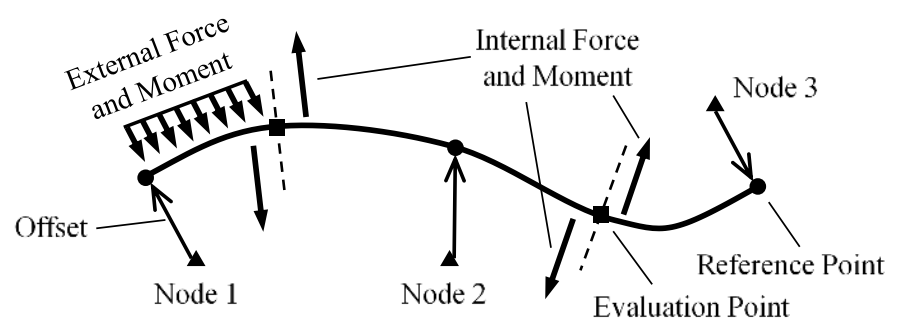

Figure 1. Illustration of a finite volume three-node beam in
MBDyn

In the application of wind turbines, modelling a turbine blade with beam elements could be justified because of the slender characteristics of the blade. As compared to conventionally used Finite Element Analysis (FEA) tools, adopting a MBD beam model for flexible blades can directly handle the arbitrary motion of an aero-elastic system, e.g. rigid body motion [6]. Given an offshore floating wind turbine case, the above functionality could cope with the rotation of the turbine, as well as the surge, heave and pitch motion of a floating platform excited by wave and wind. Furthermore, MBD simulations can be much faster than those using FEA thanks to the much fewer equations to be solved in the former approach.

\section{Mesh Motion Solver}

One of the challenges for a fully coupled numerical simulation on an offshore wind turbine with flexible blades is how to handle the computational mesh motion to represent the complex body movement involved. In addition to the turbine rotation around its hub, the flexible blades are also subject to deformation in both flapwise and edgewise directions due to the exerted unsteady wind loading. There are some commonly used moving mesh techniques, such as the sliding mesh and overset mesh, for global wind turbine rotation with rigid blades. However, they are unable to cope with the local blade deformation. It is thus necessary to develop/implement a mesh motion solver to update computational mesh both globally and 
locally. This is achieved by developing a customised mesh motion library in OpenFOAM, which incorporates the solid body motion library into the dynamic mesh deformation library. The solid body motion library can handle the global turbine rotation with the help of Arbitrary Mesh Interface (AMI), an OpenFOAM implementation of the sliding mesh technique, while the dynamic mesh library takes care of the local blade deformation.

In order to maintain the quality of the computational mesh, the global rotation is firstly subtracted from the point displacement of the FSI interface, i.e. the turbine blade surfaces. The mesh motion solver then takes the temporary point displacement of the structure boundaries as the boundary condition of the Laplace equation as shown in Eq. (6), and subsequently solves the equation for the displacement of the internal mesh cell centres [11]. Only the cells inside the rotating zone are included to reduce the computation time when the mesh motion equation is assembled. Displacement of the internal mesh points are then interpolated from cell centre displacement. The locally deformed points are lastly rotated as a whole in a manner of solid body motion to take the global rotation into consideration.

$$
\nabla \cdot\left(\gamma \nabla \mathbf{x}_{g}\right)=0
$$

where $\mathbf{x}_{g}$ is the displacement of the internal mesh cell centres; $\gamma=1 / r^{2}$ represents the diffusion coefficient and $r$ is the distance from mesh cell centres to the structure boundaries.

\section{Coupling Strategy}

The CFD solver pimpleDyMFoam and the MBD code MBDyn are coupled via motion and force data exchange with the help of a TCP/IP internet protocol. In general, the kinematics of the geometric nodes representing the bodies, specifically the position and orientation in the global reference frame calculated in MBDyn, are transferred to the CFD solver. Meanwhile, the external aerodynamic force and moment are integrated in the CFD solver over each body surface and subsequently transferred to MBDyn. Since a complete set of data exchange interfaces is available in MBDyn, only an interface class needs to be implemented in OpenFOAM. This can be achieved by wrapping the corresponding functions to receive motion from and send force to MBDyn. The interface class is then used in the mesh motion library. Apart from updating the computational mesh, the mesh motion library also serves as the bridge connecting the flow and structural solvers.

The communication between OpenFOAM and MBDyn is performed at the iteration level so that a strong coupling is achieved with robust and quick convergence. Within each time step, data is exchanged between OpenFOAM and MBDyn for a couple of times until the flow field eventually converges. MBDyn then continues its iteration with the external loading received from last data transfer until structural response approaches a converged status.

As it is mentioned earlier, a flexible wind turbine blade is represented as a series of beam elements in the MBD model, while such a blade consists of a surface mesh in the CFD solver. It is therefore necessary to establish an interpolation or mapping method to transform the CFD surface mesh with the information from the finite number of blades nodes in the MBD model. To achieve this goal, a linear interpolation scheme is currently implemented in the span-wise direction of blades while the blade cross sections are always kept flat [6].

\section{VALIDATION}

To validate the developed tool for FSI applications, a benchmark case, previously studied by many researchers $[12$, 13], is investigated where a flexible cantilever beam is attached to a rigid square cylinder in a current, as shown in Figure 2. It is noted that at a sufficiently large Reynolds number, vortices appear in the wake of cylinder, and the unsteady forces exerted on the cantilever beam would result in the oscillating motion of the beam. The boundary conditions are specified as follows. A fixed velocity of $0.513 \mathrm{~m} / \mathrm{s}$ is imposed at the left inlet boundary and zero pressure is applied at the right outlet boundary. The symmetry boundary condition is assumed at the top and bottom while an empty boundary condition, which in OpenFOAM indicates a 2D simulation, is employed for the front and back planes. The physical properties of both the fluid and solid are listed in Table 1.

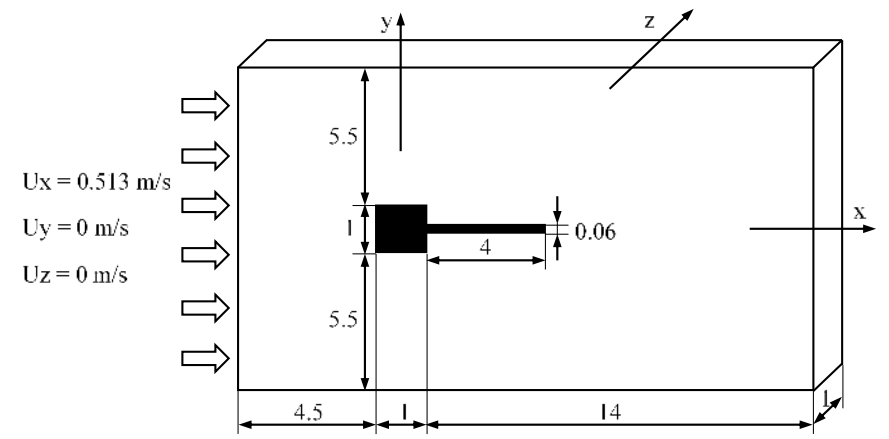

Figure 2. A flexible cantilever beam attached to a fixed rigid square in current (Dimensions in $\mathrm{cm}$ )

Table 1. Physical properties of the flexible cantilever beam

\begin{tabular}{|c|l|c|c|}
\hline \multirow{3}{*}{ Solid } & Density $\rho_{s}$ & $\mathrm{~kg} / \mathrm{m}^{3}$ & 100 \\
\cline { 2 - 4 } & Young's Modulus $E$ & $P a$ & $2.5 \mathrm{E} 5$ \\
\cline { 2 - 4 } & Poisson's Ratio & - & 0.35 \\
\hline \multirow{2}{*}{ Fluid } & Density $\rho_{f}$ & $\mathrm{~kg} / \mathrm{m}^{3}$ & 1.18 \\
\cline { 2 - 4 } & Kinematic Viscosity $v_{f}$ & $\mathrm{~m}^{2} / \mathrm{s}$ & $1.54 \mathrm{E}-5$ \\
\hline
\end{tabular}

Figure 3 demonstrates the computational mesh with a total number of 18,904 structured cells for the CFD solver and mesh refinement is deliberately applied near the solid boundaries to better capture the rapid variance of the flow field. Only one layer of grid cells is deployed in the $\mathrm{z}$ direction to constrain the fluid flow in 2D xoy plane.

In the MBD model, the flexible cantilever beam is represented by four beam elements as shown in Figure 4. Two adjacent beam elements share a same geometrical node. Since 
each beam element consists of three geometrical nodes, the total number of nodes is nine. All nodes are evenly distributed with a distance of $0.5 \mathrm{~cm}$.

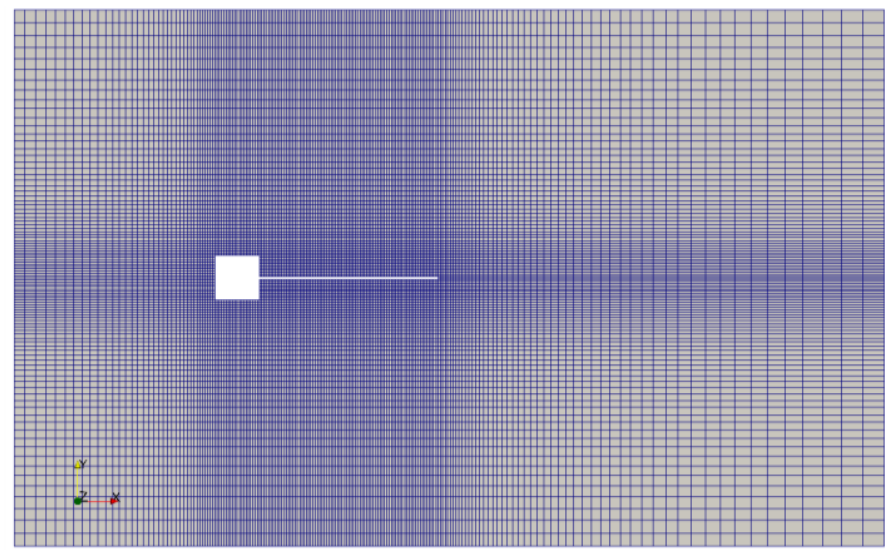

Figure 3. CFD mesh of a flexible cantilever beam used in OpenFOAM

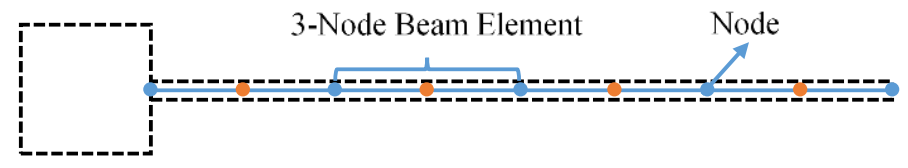

Figure 4. MBD model of flexible cantilever beam

The displacement in the y direction of the beam tip, i.e. the free end of the cantilever beam, with respect to time is plotted in Figure 5. The oscillation of the beam induced by the vortex shedding from the front cylinder gradually develops into a harmonic mode with a constant amplitude and frequency of $1.03 \mathrm{~m}$ and $3.18 \mathrm{~Hz}$, respectively. This is comparable to other available data as summarised in Table 2 , indicating that the fully coupled FSI tool developed is accurate enough.

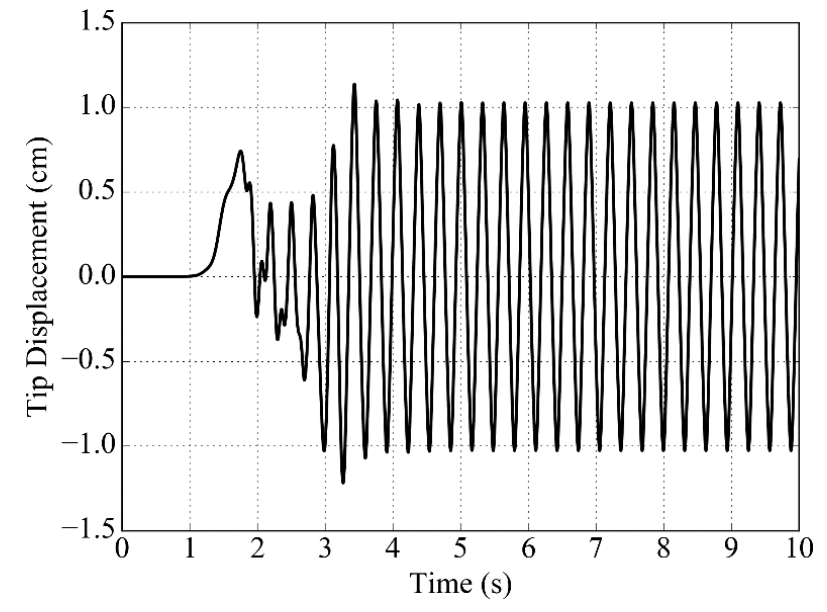

Figure 5. Tip displacement of flexible cantilever beam in y direction
Table 2. Comparison of present results with those from others' work for a flexible cantilever beam

\begin{tabular}{|l|c|c|}
\hline & $\begin{array}{c}\text { Tip Displacement } \\
\text { Amplitude (cm) }\end{array}$ & $\begin{array}{c}\text { Oscillation } \\
\text { Frequency (Hz) }\end{array}$ \\
\hline Present & 1.03 & 3.18 \\
\hline Habchi, et al. [12] & 1.02 & 3.25 \\
\hline Wood, et al. [13] & 1.15 & 2.94 \\
\hline Mathieu, et al. [14] & 0.95 & 3.17 \\
\hline $\begin{array}{l}\text { Matthies and } \\
\text { Steindorf [15] }\end{array}$ & 1.18 & 3.13 \\
\hline
\end{tabular}

Figure 6 shows the shape of the cantilever beam at different time instants represented by a series of dashed curves over one oscillation period. The trajectory of every geometrical node is also illustrated by a solid curve in a distinctive colour.

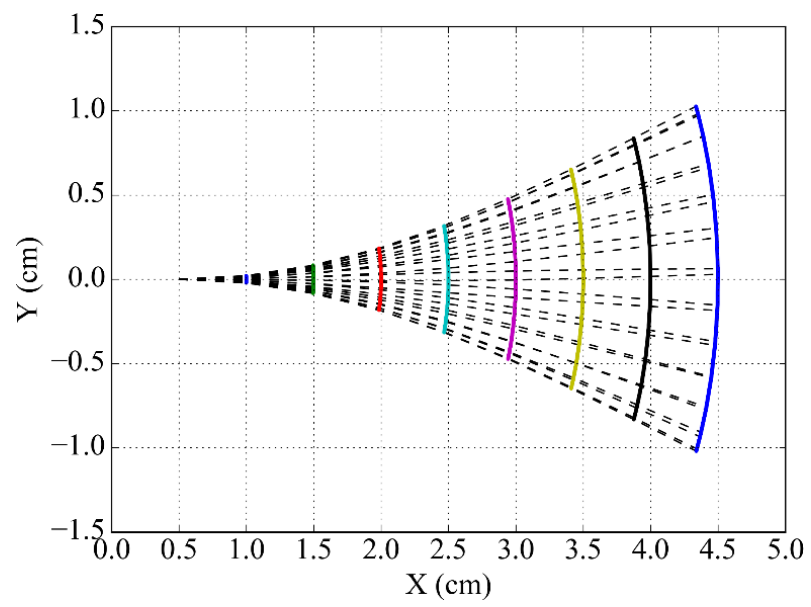

Figure 6. Shape of flexible cantilever beam over one oscillation period

Snapshots of the fluid field are also shown in Figure 7 at three subsequent time instants to demonstrate the interaction between the fluid field and the flexible cantilever beam. The $\mathrm{z}$ component of vorticity vector is used to colour the fluid field. It can be clearly seen that the deformation of the cantilever beam diverts the Von Karman vortex street induced by the front cylinder. The tip oscillation of the cantilever beam also leads to the generation of tip vortices travelling away from beam.

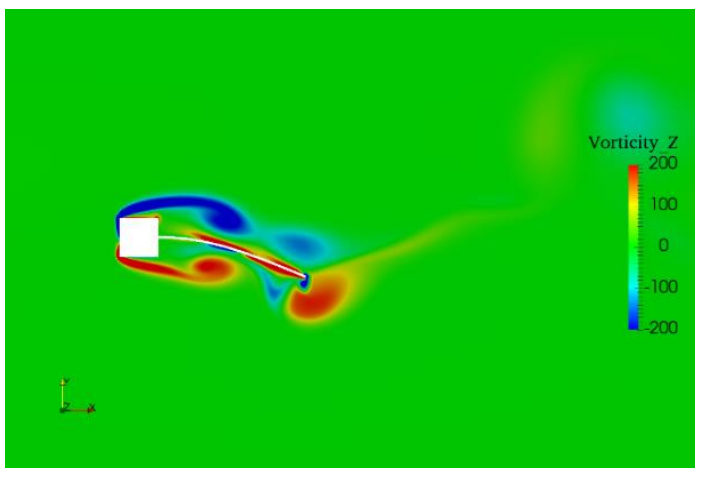

a) Time $=$ T0 


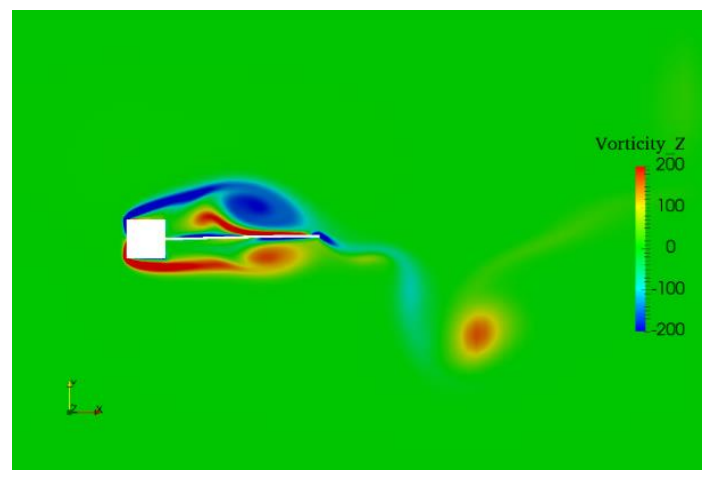

b) $\mathrm{Time}=\mathrm{T} 0+\mathrm{T} / 4$

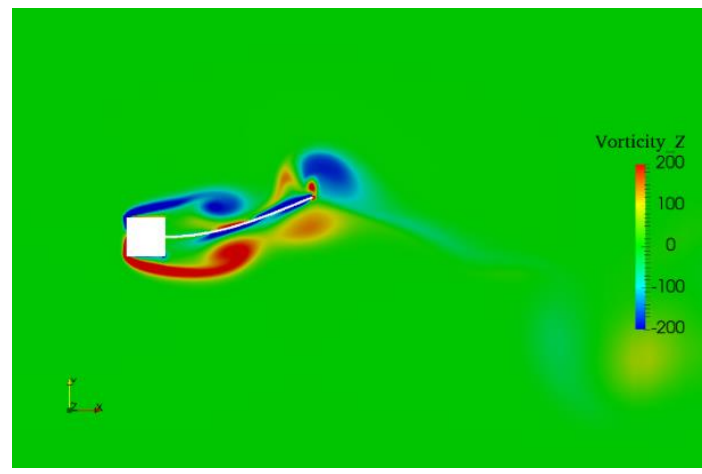

c) Time $=\mathrm{T} 0+\mathrm{T} / 2$

Figure 7. Snapshots of flow field coloured by the $z$ component of vorticity vector for a flexible cantilever beam

\section{MODEL DESCRIPTION}

The NREL 5 MW baseline offshore wind turbine [2] is then used for all subsequent simulations. It is a conventional threeblade upwind wind turbine with a rotor diameter of 126 meters, as shown in Figure 8, which is designed for offshore applications and has been widely used as a baseline model by researchers in the offshore wind energy field. Detailed information about the geometry and structural properties can be found in a report [2] published by NREL.

\section{CFD Model}

Figure 9 illustrates the dimensions of the computational domain and the boundary conditions applied in our CFD model. The domain is 700 meters long, 400 meters wide and 400 meters high. A fixed wind speed in the $\mathrm{x}$ direction is imposed at the Inlet boundary and zero pressure is applied at the Outlet boundary. The Neumann boundary condition is assumed for both velocity and pressure at the Farfield boundaries while a wall boundary condition is employed for the bottom plane. A moving wall boundary condition is applied to the turbine blades.

A grey region is highlighted in Figure 9 as the rotation cell zone. The cell zone rotates with the turbine in accordance with the specified rotation speed thus only contains the rotary parts of the turbine including the turbine blades and the hub while the nacelle and the tower are placed inside the outside grid which remains static during the simulation. The rotation region connects with the static region through a pair of AMI patches. For flexible turbine blade simulations, dynamic mesh motion due to blade deformation also occurs within the rotation cell zone.

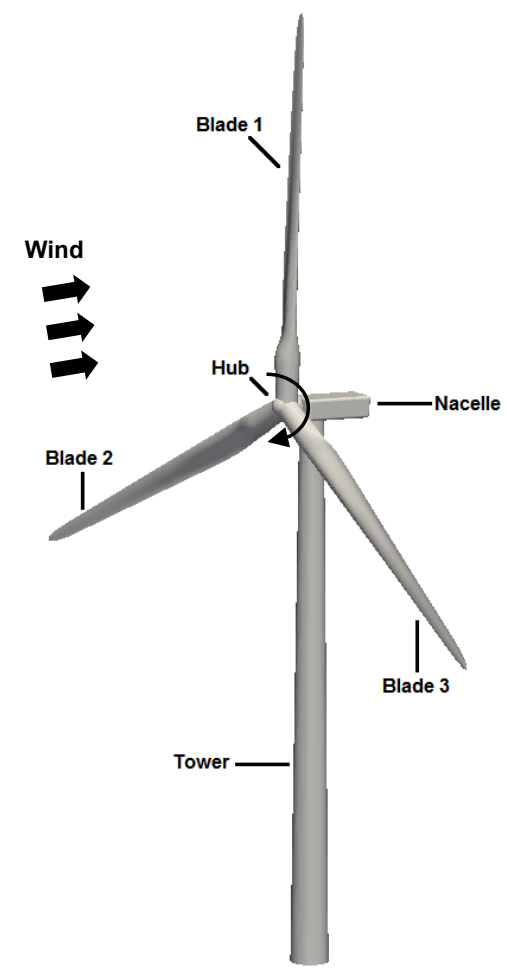

Figure 8. Geometry of the NREL $5 \mathrm{MW}$ offshore wind turbine

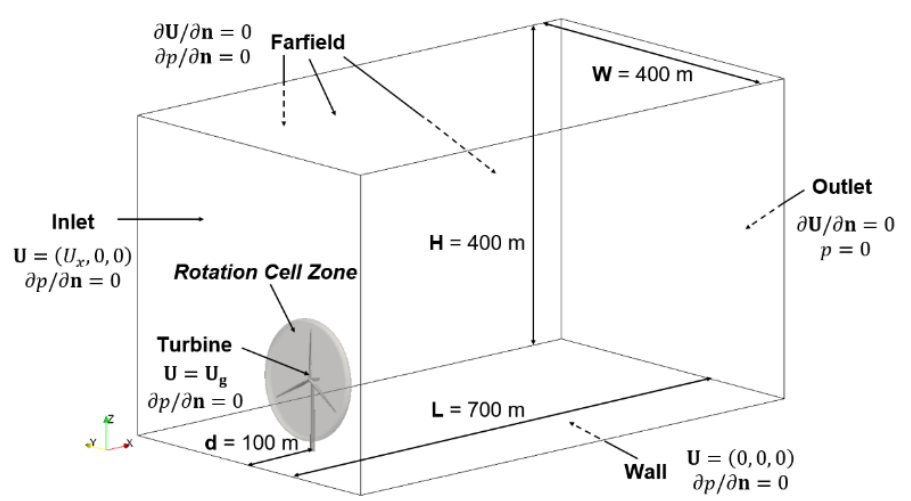

Figure 9. Dimensions and boundary conditions for NREL 5 MW offshore wind turbine simulations

Figure 10 shows the surface mesh generated with the builtin utility snappyHexMesh in OpenFOAM for the NREL $5 \mathrm{MW}$ wind turbine geometry. Mesh refinement is applied near the leading and trailing edges of the blades as well as the blade tip region to better capture the fluid flow. Eight layers of boundary layer cells are extruded from the turbine surface with a growth ratio of 1.3 and the first cell height away from the turbine surface is $0.003 \mathrm{~m}$. 


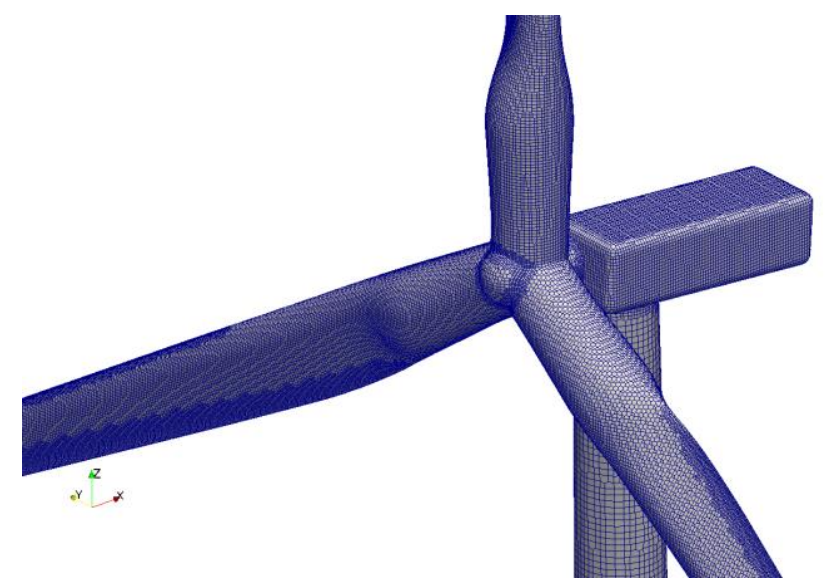

Figure 10. Surface mesh of the NREL 5 MW wind turbine

\section{Structural Model}

The structural model for the NREL $5 \mathrm{MW}$ offshore wind turbine is illustrated in Figure 11. Twelve 3-node beam elements, i.e. 25 geometrical nodes, are used to represent one blade in MBDyn. These nodes are unevenly distributed along the blade. Specifically, more nodes are assigned to the blade root and tip. The turbine hub is regarded as a rigid body thus is represented by only one node. The nacelle and tower are assumed to be rigid and fixed thus not considered in the MBD model. For a threeblade turbine, the total number of geometrical nodes is 76 . The hub node is forced to rotate with the specified turbine rotation speed using an axial rotation joint. The node at the blade root and the hub node are connected with a clamp constraint, i.e. no relative position and orientation are allowed. As a result, the blade also rotates along with the hub.

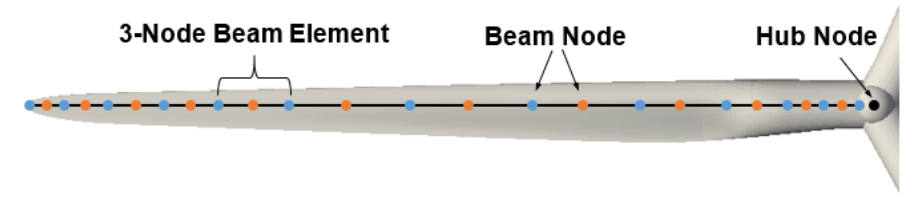

Figure 11. Structural model of the NREL $5 \mathrm{MW}$ wind turbine

\section{Simulation Cases}

Two load cases are selected from the simulation matrix of the Offshore Code Comparison Collaboration (OC3) project Phase I [16] and summarised in Table 3. The wind speed and rotational rotor speed are specified as the same as $8 \mathrm{~m} / \mathrm{s}$ and 9 RPM for both Cases 2.1a and 3.1, while Case 2.1a models rigid blades and Case 3.1 is associated with flexible blades. It is worth mentioning that, no variable rotor speed controller considered in OC3 is modelled for the Case 3.1 in this study.

Table 3. Specifications for simulation cases

\begin{tabular}{|c|c|c|}
\hline Case & DOFs & Wind Condition \\
\hline 2.1a & $\begin{array}{c}\text { Rigid blades: constant } \\
\text { rotor speed, 9 RPM }\end{array}$ & \multirow{2}{*}{ Uniform, $8 \mathrm{~m} / \mathrm{s}$} \\
\cline { 1 - 2 } 3.1 & $\begin{array}{c}\text { Flexible blades: constant } \\
\text { rotor speed, 9 RPM }\end{array}$ & \\
\hline
\end{tabular}

\section{RESULTS AND DISCUSSION}

In this part, our simulation results will be presented and compared with available data from BEM-based NREL FAST v7 in the OC3 project [16] and the CFD-based CFDShip-Iowa [7]. In particular, the blade deformation and aerodynamic performance of turbine will be fully analysed.

\section{Structural Response}

Figure 12 displays the blade deformation due to the unsteady aerodynamic loading, defined as the flapwise deflection between the rigid (grey) and flexible (blue) blades, also commonly referred to as out-of-plane deflection, in the reference frame represented by the rotor plane and rotor axis. A positive deflection points towards the downwind direction. It should be noted that there is a shaft tilt angle of $5^{\circ}$ between the rotor axis and the global $\mathrm{x}$ direction because of tower clearance and a precone angle of $2.5^{\circ}$ also exists between the blade and the rotor plane.

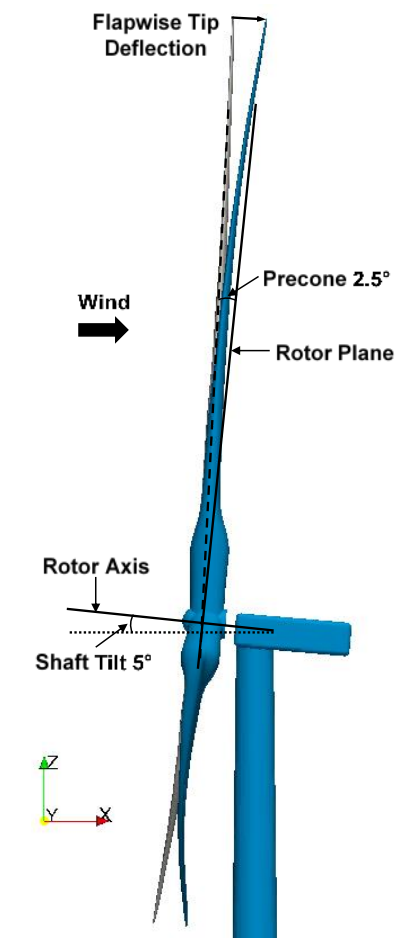

Figure 12. Definition of blade flapwise tip direction

Figure 13 shows the predicted Blade 1 tip deflection in the flapwise direction together with the results from CFDShip-Iowa and NREL FAST within two rotational periods. Similar trend can be observed for all three curves as is seen from Figure 13. At the beginning of one rotation, when Blade 1 is at the bottom of the rotor plane, the blade deflection is maximum; subsequently, a sharp drop occurs due to the interference of the tower, commonly known as the tower shadow effects, resulting in a local minimum after around 1/10 rotation. After a half rotation, the blade moves to the top of the rotor plane, and the deflection decreases to its second minimum. Further investigation is needed to understand the underlying reasons. A statistical comparison is made in Table 
4. The present simulation predicts a mean tip deflection of 3.148 $\mathrm{m}$, while CFDShip-Iowa and NREL FAST estimate a bit larger averaged values of $3.592 \mathrm{~m}$ and $3.244 \mathrm{~m}$, respectively. Considering the complexity of modelling an aero-elastic blade, the agreement among different results is still acceptable.

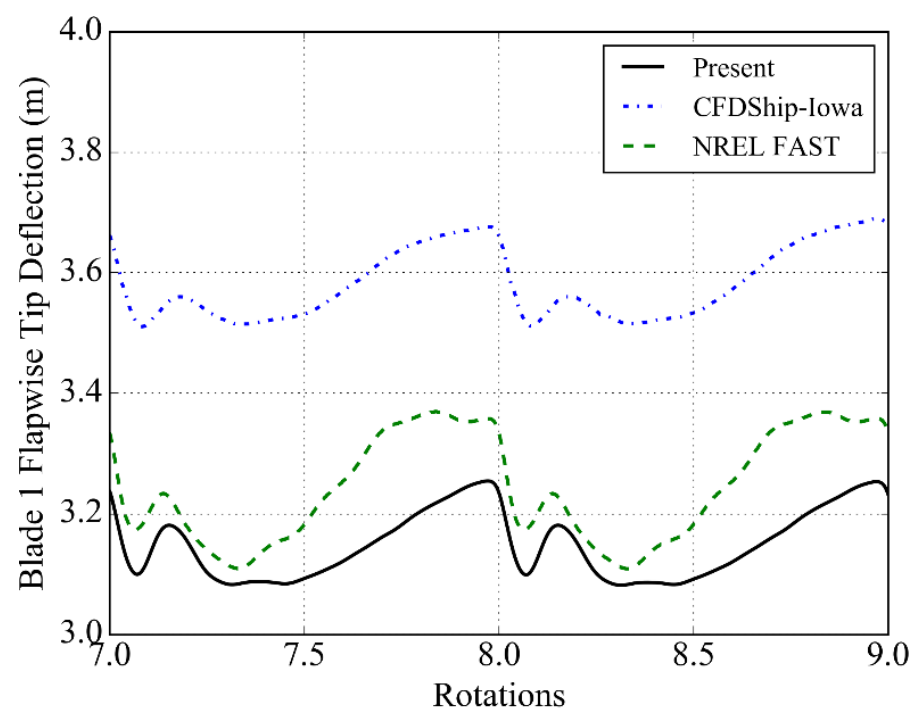

Figure 13. Blade 1 flapwise tip deflection for Case 3.1

Table 4. Comparison of Blade 1 flapwise tip deflection for Case 3.1

\begin{tabular}{|c|c|c|c|}
\hline $\begin{array}{c}\text { Flapwise Tip } \\
\text { Deflection }(\mathrm{m})\end{array}$ & Present & CFDShip-Iowa & NREL FAST \\
\hline Mean & 3.148 & 3.592 & 3.244 \\
\hline Min & 3.081 & 3.510 & 3.109 \\
\hline Max & 3.255 & 3.692 & 3.370 \\
\hline$\sigma$ & 0.056 & 0.059 & 0.090 \\
\hline
\end{tabular}

\section{Aerodynamic Performance}

In order to examine the effects of blade elasticity on the wind turbine aerodynamic performance, the thrust and torque for Cases 2.1a and 3.1 are plotted in Figure 14 and quantitatively summarised in Table 5. During one revolution, three sharp decreases are observed in both thrust and torque due to the tower shadow effects, when three blades successively pass in front of the tower. The flexible blades exhibit slightly larger thrust than rigid blades, while the predicted torques are very close regardless of the flexibility of blades. This is in accordance with CFDShipIowa simulations, but dissimilar to NREL FAST. In addition, the standard deviation of both thrust and torque from NREL FAST $(\sigma)$ decreases significantly as shown in Table 5 . It is believed that these deviations are closely linked to the variable rotor speed controller applied in NREL FAST simulations, which is not included in the present and CFDShip-Iowa modelling. It is thought that the controller is able to adapt the rotor rotation simultaneously with the incoming wind speed in order to maintain a maximal turbine power, which may result in more stable thrust and torque as compared to those without controller.
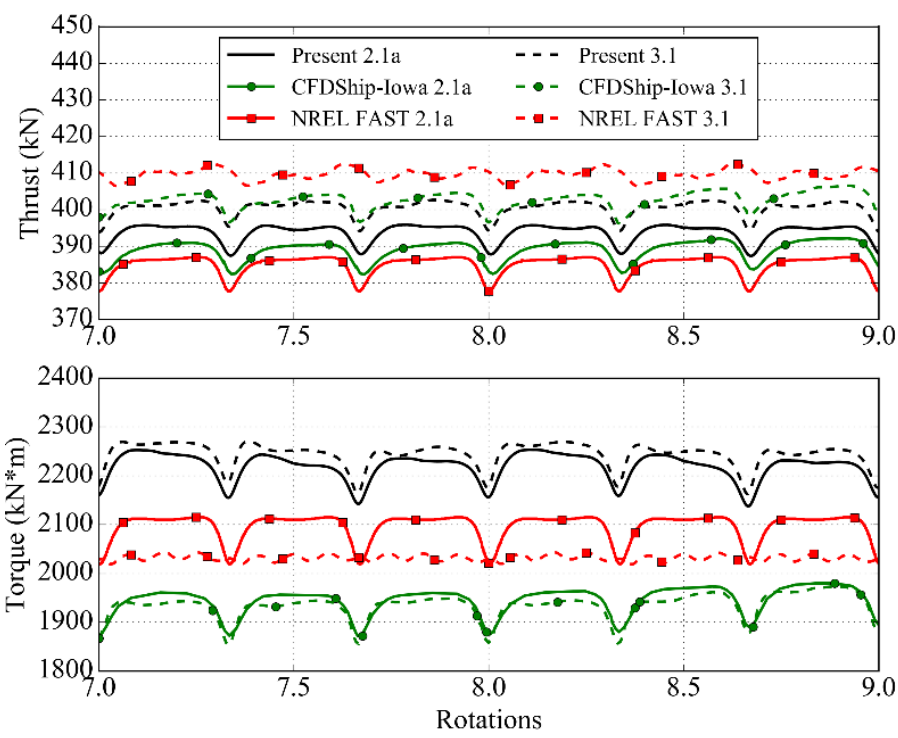

Figure 14. Turbine thrust and torque for Cases 2.1a and 3.1

Table 5. Comparison of turbine thrust and torque

\begin{tabular}{|c|c|c|c|c|c|}
\hline \multicolumn{2}{|c|}{ Cases } & \multicolumn{2}{c|}{ Thrust $(\mathrm{kN})$} & \multicolumn{2}{c|}{ Torque $(\mathrm{kN} * \mathrm{~m})$} \\
\cline { 3 - 6 } \multicolumn{2}{|c|}{} & Mean & $\sigma$ & Mean & $\sigma$ \\
\hline \multirow{2}{*}{\begin{tabular}{c} 
Present \\
\cline { 2 - 6 }
\end{tabular}} & $2.1 \mathrm{a}$ & 393.8 & 2.36 & 2218.5 & 27.05 \\
\cline { 2 - 6 } & 3.1 & 400.6 & 2.12 & 2245.2 & 24.39 \\
\hline $\begin{array}{c}\text { CFDShip- } \\
\text { Iowa }\end{array}$ & $2.1 \mathrm{a}$ & 388.9 & 2.71 & 1945.7 & 27.53 \\
\cline { 2 - 6 } & 3.1 & 402.6 & 2.30 & 1934.6 & 25.73 \\
\hline \multirow{2}{*}{$\begin{array}{c}\text { NREL } \\
\text { FAST }\end{array}$} & $2.1 \mathrm{a}$ & 385.0 & 2.60 & 2096.4 & 27.37 \\
\cline { 2 - 6 } & 3.1 & 409.6 & 1.46 & 2031.6 & 5.87 \\
\hline
\end{tabular}

Figure 15 displays the pressure distribution at three radial sections of Blade 1, i.e. $30 \%, 60 \%$ and $90 \%$ of blade radius, when the blade is at the top of the rotor plane. Both rigid and flexible simulations are plotted for the comparison. No significant differences can be observed for a flexible and rigid blade, explaining why the wind turbine thrust and torque are hardly affected by the deformation of blades.
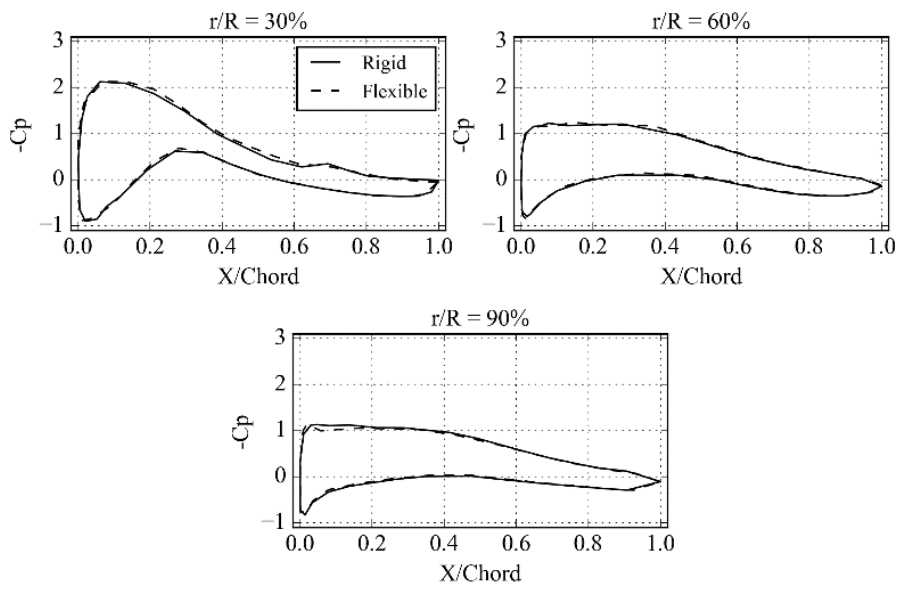

Figure 15. Pressure coefficient comparison at three different radial sections of Blade 1 at top of rotor plane 
Figure 16 illustrates the vortex contour of the second invariant of the rate of strain tensor $Q(Q=0.2)$ coloured by velocity component Ux. Clearly seen from the figure, strong vortices appear in the vicinity of the blade tips and roots. The presence of the tower also results in a complex flow wake behind the tower.

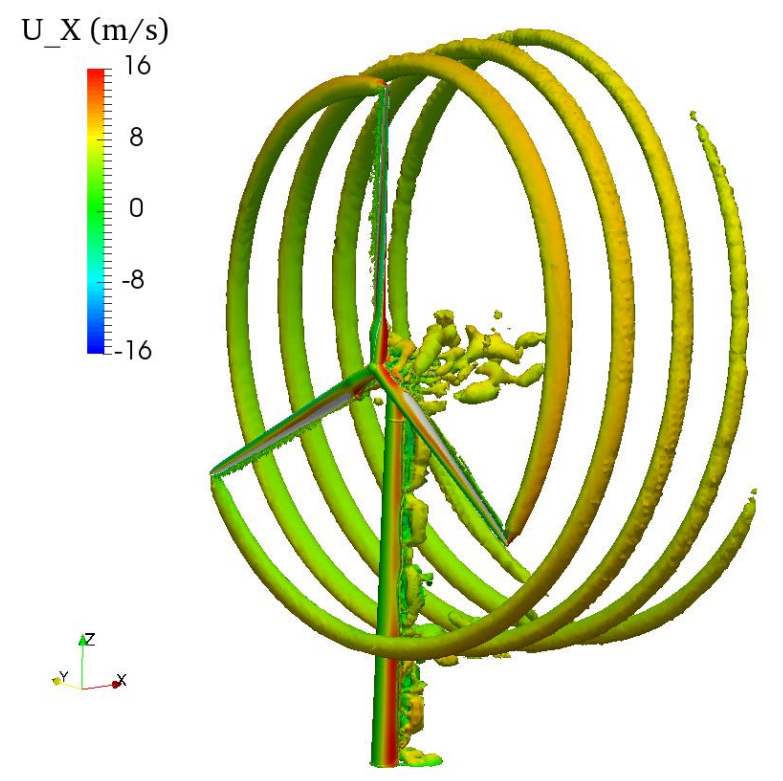

Figure 16. Vortex contour $(Q=0.2)$ coloured by velocity component Ux

\section{CONCLUSIONS}

In this paper, a fully coupled CFD/MBD analysis tool has been established for a study of offshore wind turbines. A highfidelity CFD code OpenFOAM is adopted for solving the fluid flow while a MultiBody Dynamics code MBDyn is applied for predicting the flexible structural response. A customised mesh motion solver is developed to handle moving mesh involving both global turbine rotation and localised blade deformation. This solver also serves as the bridge connecting the flow and structural solvers.

A benchmark case of a flexible cantilever beam subject to the vortices in the wake of a fixed square cylinder is firstly investigated to validate the analysis tool. The predicted oscillating amplitude and frequency of the tip displacement are compared with previous results, and good agreement is achieved.

The NREL 5 MW baseline offshore wind turbine is subsequently modelled with both rigid and flexible blades using the developed CFD/MBD analysis tool. Modelling results are analysed and compared with publicly available data from Iowa CFD code and BEM-based NREL FAST. Our predicted timemean blade flapwise tip deflection of $3.15 \mathrm{~m}$ is in good agreement with others. Due to the exclusion of a variable rotor speed controller in the CFD modelling, the turbine thrust and torque obtained by two CFD tools (Iowa CFD and the present CFD), are not much affected by the blade deformation, which is justified by the almost identical pressure distribution at three blade spanwise sections for both a rigid and flexible blade. The good agreement achieved between the present results and data obtained using Iowa CFD and FAST has demonstrated and verified the capability of our tool. The proposed approach will then be extended to investigate floating offshore wind turbines with deformable blades under more complex environmental conditions, where FAST might be insufficient.

Our near future study will move onto a floating offshore wind turbine with aero-elastic blades. The effect of floating platform on the wind turbine power will be firstly investigated with a prescribed platform motion. Development of a fully coupled aero-hydro-mooring-elastic CFD tool is our undergoing task. With this tool, we will be able to model a floating offshore wind turbine with flexible blades plus the relevant mooring systems under combined wind-wave conditions.

\section{ACKNOWLEDGMENTS}

Results were obtained using the EPSRC funded ARCHIEWeSt High Performance Computer (www.archie-west.ac.uk). EPSRC grant No. EP/K000586/1. This work is also supported by EPSRC RAP project for accessing ARCHER UK National Supercomputing Service (http://www.archer.ac.uk). The first author would like to acknowledge Électricité de France for providing the scholarship for his $\mathrm{PhD}$ research.

\section{REFERENCES}

[1] WindEurope, 2016, "The European offshore wind industry: Key trends and statistics 1st half 2016."

[2] Jonkman, J. M., Butterfield, S., Musial, W., and Scott, G., 2009, "Definition of a 5-MW reference wind turbine for offshore system development," No. NREL/TP-500-38060, National Renewable Energy Laboratory, Golden, Colorado, USA.

[3] Jonkman, J. M., 2007, "Dynamics modeling and loads analysis of an offshore floating wind turbine," $\mathrm{PhD}$ in Aerospace Engineering Doctoral Dissertation, University of Colorado at Boulder, Boulder, Colorado.

[4] Carrión, M., Steijl, R., Woodgate, M., Barakos, G. N., Munduate, X., and Gomez-Iradi, S., 2014, "Aeroelastic analysis of wind turbines using a tightly coupled CFD-CSD method," Journal of Fluids and Structures, 50, pp. 392-415.

[5] Yu, D. O., and Kwon, O. J., 2014, "Predicting wind turbine blade loads and aeroelastic response using a coupled CFD-CSD method," Renewable Energy, 70, pp. 184-196.

[6] Pierangelo, M., and Jayanarayanan, S., "Coupled CFD/Multibody Analysis of NREL Unsteady Aerodynamic Experiment Phase VI Rotor," Proc. 49th AIAA Aerospace Sciences Meeting including the New Horizons Forum and Aerospace Exposition, American Institute of Aeronautics and Astronautics.

[7] Li, Y., Castro, A. M., Sinokrot, T., Prescott, W., and Carrica, P. M., 2015, "Coupled multi-body dynamics and CFD for wind turbine simulation including explicit wind turbulence," Renewable Energy, 76, pp. 338-361.

[8] Heinz, J. C., Sørensen, N. N., Zahle, F., and Skrzypiński, W., 2016, "Vortex-induced vibrations on a modern wind turbine blade," Wind Energy, 19(11), pp. 2041-2051. 
[9] Menter, F. R., 2009, "Review of the shear-stress transport turbulence model experience from an industrial perspective," International Journal of Computational Fluid Dynamics, 23(4), pp. 305-316.

[10] Ghiringhelli, G. L., Masarati, P., and Mantegazza, P., 2000, "Multibody Implementation of Finite Volume C Beams," AIAA Journal, 38(1), pp. 131-138.

[11] Jasak, H., and Tukovic, Z., 2006, "Automatic mesh motion for the unstructured finite volume method," Transactions of FAMENA, 30(2), pp. 1-20.

[12] Habchi, C., Russeil, S., Bougeard, D., Harion, J.-L., Lemenand, T., Ghanem, A., Valle, D. D., and Peerhossaini, H., 2013, "Partitioned solver for strongly coupled fluid-structure interaction," Computers \& Fluids, 71, pp. 306-319.

[13] Wood, C., Gil, A. J., Hassan, O., and Bonet, J., 2010, "Partitioned block-Gauss-Seidel coupling for dynamic fluid- structure interaction," Computers \& Structures, 88(23-24), pp. 1367-1382.

[14] Mathieu, O., Jean-Francois, M., and Guy, D., 2009, "A Fluid-Structure Interaction Solver for Nano-Air-Vehicle Flapping Wings," 19th AIAA Computational Fluid Dynamics, American Institute of Aeronautics and Astronautics.

[15] Matthies, H. G., and Steindorf, J., 2003, "Partitioned strong coupling algorithms for fluid-structure interaction," Computers \& Structures, 81(8-11), pp. 805-812.

[16] Jonkman, J., and Musial, W., 2010, "Offshore Code Comparison Collaboration (OC3) for IEA Task 23 Offshore Wind Technology and Deployment," No. NREL/TP-500048191, National Renewable Energy Laboratory, Golden, Colorado, USA. 